\title{
Some identities on the twisted $q$-Bernoulli numbers and polynomials with weight $\alpha$
}

\section{Lee-Chae Jang*}

"Correspondence:

leechae.jang@kku.ac.kr

Department of Mathematics and

Computer Science, Kon-Kuk

University, Chungju, 138-701, Korea

\begin{abstract}
In this paper, we consider the twisted $q$-Bernoulli numbers and polynomials with weight $\alpha$ by using the bosonic $q$-integral on $\mathbb{Z}_{p}$. From the construction of the twisted $q$-Bernoulli numbers with weight $\alpha$, we derive some identities and relations.

MSC: Primary 11B68; 11S40; 11S80
\end{abstract}

Keywords: Bernoulli numbers and polynomials; bosonic q-integral; twisted $q$-Bernoulli numbers and polynomials with weight $\alpha$

\section{Introduction}

Let $p$ be a fixed prime number. Throughout this paper, $\mathbb{Z}_{p}, \mathbb{Q}_{p}$, and $\mathbb{C}_{p}$ will, respectively, denote the ring of $p$-adic rational integers, the field of $p$-adic rational numbers, and the $p$-adic completion of the algebraic closure of $\mathbb{Q}_{p}$, respectively. Let $v_{p}$ be the normalized exponential valuation of $\mathbb{C}_{p}$ with $|p|_{p}=p^{v_{p}(p)}=p^{-1}$. When one talks of $q$-extension, $q$ is variously considered as an indeterminate, a complex number $q \in \mathbb{C}$, or a $p$-adic number $q \in \mathbb{C}_{p}$. If $q \in \mathbb{C}$, one normally assume $|q|<1$. If $q \in \mathbb{C}_{p}$, then we assume that $|1-q|_{p}<p^{-\frac{1}{1-p}}$, so that $q^{x}=\exp (x \log q)$ for $|x|_{p} \leq 1$. The $q$-number is defined by $[x]_{q}=\frac{1-q^{x}}{1-q}$ (see [1-25]). Note that $\lim _{q \rightarrow 1}[x]_{q}=x$.

We say that $f$ is uniformly differentiable function at a point $a \in \mathbb{Z}_{p}$, and denote this property by $f \in U D\left(\mathbb{Z}_{p}\right)$, if the difference quotient $F_{f}(x, y)=\frac{f(x)-f(y)}{x-y}$ has a limit $f^{\prime}(a)$ as $(x, y) \rightarrow(a, a)$. For $f \in U D\left(\mathbb{Z}_{p}\right)$, the $p$-adic $q$-integral on $\mathbb{Z}_{p}$, which is called the bosonic $q$-integral, defined by Kim as follows:

$$
I_{q}(f)=\int_{\mathbb{Z}_{p}} f(x) d \mu_{q}(x)=\lim _{N \rightarrow \infty} \frac{1}{\left[p^{N}\right]_{q}} \sum_{x=0}^{p^{N}-1} f(x) q^{x} \quad(\text { see [14]). }
$$

From (1), we note that

$$
\left|\int_{\mathbb{Z}_{p}} f(x) d \mu_{q}(x)\right|_{p} \leq p\|f\|_{1} \quad(\text { see }[11-15]),
$$

where $\|f\|_{1}=\sup \left\{|f(0)|_{p}, \sup _{x \neq y}\left|\frac{f(x)-f(y)}{x-y}\right|_{p}\right\}$.

\section{Springer}

(c) 2012 Jang: licensee Springer. This is an Open Access article distributed under the terms of the Creative Commons Attribution License (http://creativecommons.org/licenses/by/2.0), which permits unrestricted use, distribution, and reproduction in any medium, provided the original work is properly cited. 
In [3], Carlitz defined $q$-Bernoulli numbers which are called the Carlitz's $q$-Bernoulli numbers, by

$$
\beta_{0, q}=1 \quad \text { and } q\left(q \beta_{q}+1\right)^{n}-\beta_{n, q}= \begin{cases}1 & \text { if } m=1 \\ 0 & \text { if } m>1\end{cases}
$$

with the usual convention about replacing $\left(\beta_{q}^{(h)}\right)^{n}$ by $\beta_{n, q}^{(h)}$. In [3], Carlitz also considered the expansion of $q$-Bernoulli numbers as follows:

$$
\beta_{0, q}^{(h)}=\frac{h}{[h]_{q}} \quad \text { and } \quad q^{h}\left(q \beta_{q}^{(h)}+1\right)^{n}-\beta_{n, q}^{(h)}= \begin{cases}1 & \text { if } n=1 \\ 0 & \text { if } n>1\end{cases}
$$

with the usual convention about replacing $\left(\beta_{q}^{(h)}\right)^{n}$ by $\beta_{n, q}^{(h)}$. In [15], for $\alpha \in \mathbb{Q}$ and $n \in \mathbb{Z}_{+}$, $q$-Bernoulli numbers with weight $\alpha$ is defined by Kim as follows:

$$
\beta_{0, q}^{(\alpha)} \quad \text { and } \quad q\left(q^{\alpha} \widetilde{\beta}_{q}^{(\alpha)}+1\right)^{n}-\widetilde{\beta}_{n, q}^{(\alpha)}= \begin{cases}\frac{\alpha}{[\alpha]_{q}} & \text { if } n=1 \\ 0 & \text { if } n>1\end{cases}
$$

with the usual convention about replacing $\left(\beta_{q}^{(\alpha)}\right)^{n}$ by $\beta_{n, q}^{(\alpha)}$.

Let $C_{p^{n}}=\left\{\xi \mid \xi^{p^{n}}=1\right\}$ be the cyclic group of order $p^{n}$, and let $T_{p}=\lim _{n \rightarrow \infty} C_{p^{n}}=\bigcup_{n \geq 0} C_{p^{n}}$ (see $[8,13,20-24])$. Note that $T_{p}$ is a locally constant space. For $\xi \in T_{p}$, the twisted Bernoulli numbers are defined by

$$
\frac{t}{\xi e^{t}-1}=e^{B_{\xi} t}=\sum_{n=0}^{\infty} B_{n, \xi} \frac{t^{n}}{n !},
$$

with the usual convention about replacing $\left(B_{\xi}\right)^{n}$ by $B_{n, q}($ see $[15,19-24])$.

In the view point of (6), we will try to study the twisted $q$-Bernoulli numbers with weight $\alpha$. By using the $p$-adic $q$-integral on $\mathbb{Z}_{p}$, we give some identities and relations on the twisted $q$-Bernoulli numbers and polynomials with weight $\alpha$.

\section{The twisted $q$-Bernoulli numbers and polynomials with weight $\alpha$}

Let $f_{n}(x)=f(x+n)$. In [15, Theorem 1], Kim proved the following integral equation:

$$
q^{n} I_{q}\left(f_{n}\right)-I_{q}(f)=(q-1) \sum_{l=0}^{n-1} q^{l} f(l)+\frac{q-1}{\log q} \sum_{l=0}^{n-1} q^{l} f^{\prime}(l) .
$$

In particular, when $n=1$, we have

$$
q I_{q}\left(f_{1}\right)-I_{q}(f)=(q-1) f(0)+\frac{q-1}{\log q} f^{\prime}(0) .
$$

Let $\alpha \in \mathbb{Q}$ and $n \in \mathbb{Z}_{+}$. The $n$th $q$-Bernoulli polynomials with weight $\alpha$ are defined by

$$
\widetilde{\beta}_{n, \xi, q}^{\alpha}(x)=\int_{\mathbb{Z}_{p}} \xi^{y}[y+x]_{q^{\alpha}}^{n} d \mu_{q}(y)
$$


Then, by using the bosonic $p$-adic $q$-integral on $\mathbb{Z}_{p}$, we evaluate the above equation (9) as follows:

$$
\begin{aligned}
\widetilde{\beta}_{n, \xi, q}^{\alpha}(x) & =\int_{\mathbb{Z}_{p}} \xi^{y}[y+x]_{q^{\alpha}}^{n} d \mu_{q}(y) \\
& =\frac{1}{\left(1-q^{\alpha}\right)^{n}} \sum_{l=0}^{n}\left(\begin{array}{l}
n \\
l
\end{array}\right)(-1)^{l} q^{\alpha l x} \int_{\mathbb{Z}_{p}} \xi^{y} q^{\alpha l y} d \mu_{q}(y) \\
& =\frac{1-q}{\left(1-q^{\alpha}\right)^{n}} \sum_{l=0}^{n}\left(\begin{array}{l}
n \\
l
\end{array}\right)(-1)^{l}\left(\frac{\alpha l+1}{1-\xi q^{\alpha l+1}}\right) q^{\alpha l x} .
\end{aligned}
$$

In the special case $x=0, \widetilde{\beta}_{n, \xi, q}^{\alpha}(0)=\widetilde{\beta}_{n, \xi, q}^{\alpha}$ are called the $n$th twisted $q$-Bernoulli numbers with weight $\alpha$.

From (10), we note that

$$
\begin{aligned}
\widetilde{\beta}_{n, \xi, q}^{\alpha}(x) & \\
= & \frac{1-q}{\left(1-q^{\alpha}\right)^{n}} \sum_{l=0}^{n}\left(\begin{array}{c}
n \\
l
\end{array}\right)(-1)^{l} q^{\alpha l x}\left(\frac{\alpha l}{1-\xi q^{\alpha l+1}}\right) \\
& +\frac{1-q}{\left(1-q^{\alpha}\right)^{n}} \sum_{l=0}^{n}\left(\begin{array}{c}
n \\
l
\end{array}\right)(-1)^{l} q^{\alpha l x}\left(\frac{1}{1-\xi q^{\alpha l+1}}\right) \\
= & \frac{n \alpha(1-q)}{\left(1-q^{\alpha}\right)^{n}} \sum_{l=1}^{n}\left(\begin{array}{c}
n-1 \\
l-1
\end{array}\right)(-1)^{l}\left(\frac{1}{1-\xi q^{\alpha l+1}}\right) \\
& +\sum_{m=0}^{\infty}(1-q) \frac{\left(1-q^{\alpha(x+m)}\right)^{n}}{\left(1-q^{\alpha}\right)^{n}} \xi^{m} q^{m} \\
= & -n \alpha \frac{(1-q)}{\left(1-q^{\alpha}\right)^{n}} \sum_{m=0}^{\infty} \xi^{m} q^{m \alpha+m}\left(1-q^{\alpha(x+m)}\right)^{n-1} \\
& +(1-q) \sum_{m=0}^{\infty}[x+m]_{q^{\alpha}}^{n} \xi^{m} q^{m} \\
= & \frac{-n \alpha}{[\alpha]_{q}} \sum_{m=0}^{\infty} \xi^{m} q^{m \alpha+m}[x+m]_{q^{\alpha}}^{n-1}+(1-q) \sum_{m=0}^{\infty} \xi^{m} q^{m}[x+m]_{q^{\alpha}}^{n} .
\end{aligned}
$$

From (11), when $x=0$, we get

$$
\widetilde{\beta}_{n, \xi, q}^{\alpha}=\frac{-n \alpha}{[\alpha]_{q}} \sum_{m=0}^{\infty} \xi^{m} q^{m \alpha+m}[m]_{q^{\alpha}}^{n-1}+(1-q) \sum_{m=0}^{\infty} \xi^{m} q^{m}[m]_{q^{\alpha}}^{n} .
$$

Therefore, by (10) and (12), we obtain the following theorem.

Theorem 1 Let $n \in \mathbb{N}$ and $\alpha \in \mathbb{C}$. Then we

$$
\begin{aligned}
- & \frac{\widetilde{\beta}_{n, \xi, q}^{\alpha}(x)}{n} \\
& =\frac{\alpha}{[\alpha]_{q}} \sum_{m=0}^{\infty} \xi^{m} q^{m \alpha+m}[x+m]_{q^{\alpha}}^{n-1}+\frac{1-q}{n} \sum_{m=0}^{\infty} \xi^{m} q^{m}[x+m]_{q^{\alpha}}^{n} .
\end{aligned}
$$


When $x=0$, we can obtain some identity on the twisted $q$-Bernoulli numbers with weight $\alpha$ as follows.

Corollary 2 For $n \in \mathbb{Z}_{+}$and $\alpha \in \mathbb{Q}$. Then we have

$$
-\frac{\widetilde{\beta}_{n, \xi, q}^{(\alpha)}}{n}=\frac{\alpha}{[\alpha]_{q}} \sum_{m=0}^{\infty} \xi^{m} q^{m \alpha+m}[m]_{q^{\alpha}}^{n-1}+\frac{1-q}{n} \sum_{m=0}^{\infty} \xi^{m} q^{m}[m]_{q^{\alpha}}^{n} .
$$

For $\alpha=1$, we note that $\widetilde{\beta}_{n, \xi, q}^{(1)}(x)$ are the twisted Carlitz's $q$-Bernoulli polynomials and $\widetilde{\beta}_{n, \xi, q}^{(1)}$ are the twisted Carlitz's $q$-Bernoulli numbers. By Corollary 2 , we easily get

$$
\begin{aligned}
& \int_{\mathbb{Z}_{p}} \xi^{x} e^{[x]_{q^{\alpha}} t} d \mu_{q}(x) \\
& \quad=-t \frac{\alpha}{[\alpha]_{q}} \sum_{m=0}^{\infty} \xi^{m} q^{(m+1) \alpha+m} e^{[m]_{q}^{\alpha} t}+(1-q) \sum_{m=0}^{\infty} \xi^{m} q^{m} e^{[m]_{q^{\alpha}} t} .
\end{aligned}
$$

From (13), we have

$$
\sum_{n=0}^{\infty} \widetilde{\beta}_{n, \xi, q}^{(\alpha)} \frac{t^{n}}{n !}=-t \frac{\alpha}{[\alpha]_{q}} \sum_{m=0}^{\infty} \xi^{m} q^{(m+1) \alpha+m} e^{[m] q^{\alpha} t}+(1-q) \sum_{m=0}^{\infty} \xi^{m} q^{m} e^{[m]_{q}^{\alpha} t} .
$$

Therefore, we obtain the following corollary.

Corollary 3 Let $\alpha \in \mathbb{Q}$ and $F_{\xi, q}^{(\alpha)}(t)=\sum_{n=0}^{\infty} \widetilde{\beta}_{n, \xi, q}^{(\alpha)} \frac{t^{n}}{n !}$. Then we have

$$
F_{\xi, q}^{(\alpha)}(t)=-t \frac{\alpha}{[\alpha]_{q}} \sum_{m=0}^{\infty} \xi^{m} q^{(m+1) \alpha+m} e^{[m]_{q^{\alpha}} t}+(1-q) \sum_{m=0}^{\infty} \xi^{m} q^{m} e^{[m]_{q} \alpha t}
$$

Let $F_{\xi, q}^{(\alpha)}(t, x)=\sum_{n=0}^{\infty} \sum_{n=0}^{\infty} \widetilde{\beta}_{n, \xi, q}^{(\alpha)}(x) \frac{t^{n}}{n !}$. From Theorem 1, we have

$$
\begin{aligned}
& F_{\xi, q}^{(\alpha)}(t, x) \\
& =-t \frac{\alpha}{[\alpha]_{q}} \sum_{m=0}^{\infty} \xi^{m} q^{(m+1) \alpha+m} e^{[m+x] q^{\alpha} t}+(1-q) \sum_{m=0}^{\infty} \xi^{m} q^{m} e^{[m+x]_{q^{\alpha}} t} \\
& =\sum_{m=0}^{\infty} \widetilde{\beta}_{n, \xi, q}^{(\alpha)}(x) \frac{t^{n}}{n !} .
\end{aligned}
$$

By simple calculation, we easily get

$$
\begin{aligned}
\widetilde{\beta}_{n, \xi, q}^{(\alpha)}(x) & =\int_{\mathbb{Z}_{p}} \xi^{y}[x+y]_{q^{\alpha}} d \mu_{q}(y) \\
& =\sum_{l=0}^{n}\left(\begin{array}{l}
n \\
l
\end{array}\right)[x]_{q^{\alpha}}^{n-l} q^{\alpha l x} \int_{\mathbb{Z}_{p}} \xi^{y}[y]_{q^{\alpha}}^{l} d \mu_{q}(y) \\
& =\sum_{l=0}^{n}\left(\begin{array}{l}
n \\
l
\end{array}\right)[x]_{q^{\alpha}}^{n-l} q^{\alpha l x} \widetilde{\beta}_{l, \xi, q^{*}}^{(\alpha)}
\end{aligned}
$$


Therefore, by (12), (15), and (16), we obtain the following theorem.

Theorem 4 For $n \in \mathbb{Z}_{+}$and $\alpha \in \mathbb{Q}$, we have

$$
\begin{aligned}
\widetilde{\beta}_{n, \xi, q}^{(\alpha)}(x)= & \frac{1-q}{\left(1-q^{\alpha}\right)^{n}} \sum_{l=0}^{n}\left(\begin{array}{c}
n \\
l
\end{array}\right)(-1)^{l} q^{\alpha l x}\left(\frac{\alpha l+1}{1-\xi q^{\alpha l+1}}\right) \\
= & -n \frac{\alpha}{[\alpha]_{q}} \sum_{m=0}^{\infty} \xi^{m} q^{(m+1) \alpha+m}[m+x]_{q^{\alpha}}^{n-1} \\
& +(1-q) \sum_{m=0}^{\infty} \xi^{m} q^{m}[m+x]_{q^{\alpha}}^{n} .
\end{aligned}
$$

Moreover, $\widetilde{\beta}_{n, \xi, q}^{(\alpha)}(x)=\sum_{l=0}^{n}\left(\begin{array}{l}n \\ l\end{array}\right)[x]_{q^{\alpha}}^{n-l} q^{\alpha l x} \widetilde{\beta}_{l, \xi, q^{*}}^{(\alpha)}$

By (7), we see that

$$
\begin{aligned}
& \xi^{n} q^{n} \widetilde{\beta}_{m, \xi, q}^{(\alpha)}(n)-\widetilde{\beta}_{m, \xi, q}^{(\alpha)} \\
& \quad=(q-1) \sum_{l=0}^{n-1} \xi^{l} q^{\alpha l}[l]_{q^{\alpha}}^{m}+m \frac{\alpha}{[\alpha]_{q}} \sum_{l=0}^{n-1} \xi^{l} q^{\alpha l+l}[l]_{q^{\alpha}}^{m-1} .
\end{aligned}
$$

Therefore, we obtain the following theorem.

Theorem 5 For $n \in \mathbb{Z}_{+}, n \in \mathbb{N}$, and $\alpha \in \mathbb{Q}$, we have

$$
\begin{aligned}
& \xi^{n} q^{n} \widetilde{\beta}_{m, \xi, q}^{(\alpha)}(n)-\widetilde{\beta}_{m, \xi, q}^{(\alpha)} \\
& \quad=(q-1) \sum_{l=0}^{n-1} \xi^{l} q^{\alpha l}[l]_{q^{\alpha}}^{m}+m \frac{\alpha}{[\alpha]_{q}} \sum_{l=0}^{n-1} \xi^{l} q^{\alpha l+l}[l]_{q^{\alpha}}^{m-1} .
\end{aligned}
$$

If we take $f(x)=\xi^{x} e^{[x]_{q^{\alpha}} t}$, by (8), then we have

$$
\begin{aligned}
& (q-1)+\frac{\alpha}{[\alpha]_{q}} t \\
& =q \int_{\mathbb{Z}_{p}} \xi^{x+1} e^{[x+1]_{q^{\alpha}} t} d \mu_{q}(x)-\int_{\mathbb{Z}_{p}} \xi^{x} e^{[x]_{q^{\alpha}} t} d \mu_{q}(x) \\
& =\xi q \int_{\mathbb{Z}_{p}} \xi^{x} e^{[x+1]_{q^{\alpha}} t} d \mu_{q}(x)-\int_{\mathbb{Z}_{p}} \xi^{x} e^{[x]_{q^{\alpha}} t} d \mu_{q}(x) \\
& =\sum_{n=0}^{\infty}\left(\xi q \widetilde{\beta}_{n, \xi, q}^{(\alpha)}(1)-\widetilde{\beta}_{n, \xi, q}^{(\alpha)}\right) \frac{t^{n}}{n !} .
\end{aligned}
$$

Therefore, by Theorem 5 , we obtain the following theorem.

Theorem 6 For $\alpha \in \mathbb{Q}$ and $n \in \mathbb{Z}_{+}$, we have

$$
\xi q \widetilde{\beta}_{0, \xi, q}^{(\alpha)}(1)-\widetilde{\beta}_{0, \xi, q}^{(\alpha)}= \begin{cases}q-1 & \text { if } n=0 \\ \frac{\alpha}{[\alpha]_{q}} & \text { if } n=1 \\ 0 & \text { if } n>1\end{cases}
$$


Remark that when $n=0$, we have $\widetilde{\beta}_{0, \xi, q}^{(\alpha)}=\frac{q-1}{\xi q-1}$. By (16) and Theorem 6 , we get

$$
\begin{aligned}
\widetilde{\beta}_{n, \xi, q}^{(\alpha)}(x) & =\sum_{l=0}^{n}\left(\begin{array}{l}
n \\
l
\end{array}\right)[x]_{q^{\alpha}}^{n-l} q^{\alpha l x} \widetilde{\beta}_{l, \xi, q}^{(\alpha)} \\
& =\left([x]_{q^{\alpha}}+q^{\alpha x} \widetilde{\beta}_{\xi, q}^{(\alpha)}\right)^{n},
\end{aligned}
$$

with the usual convention about replacing $\left(\widetilde{\beta}_{\xi, q}^{(\alpha)}\right)^{n}$ by $\widetilde{\beta}_{n, \xi, q}^{(\alpha)}$. By $(20)$ and Theorem 6 , we obtain the following theorem.

Theorem 7 For $n \in \mathbb{Z}_{+}$and $\alpha \in \mathbb{Q}$, we have

$$
\xi q\left(q^{\alpha} \widetilde{\beta}_{\xi, q}^{(\alpha)}+1\right)^{n}-\widetilde{\beta}_{n, \xi, q}^{(\alpha)}= \begin{cases}q-1 & \text { if } n=0 \\ \frac{\alpha}{[\alpha]_{q}} & \text { if } n=1 \\ 0 & \text { if } n>1\end{cases}
$$

with the usual convention about replacing $\left(\widetilde{\beta}_{\xi, q}^{(\alpha)}\right)^{n}$ by $\widetilde{\beta}_{n, \xi, q^{*}}^{(\alpha)}$

From (8), we can easily derive the following equation (21). For $d \in \mathbb{N}$, we get

$$
\begin{aligned}
\int_{\mathbb{Z}_{p}} \xi^{y}[x+y]_{q^{\alpha}}^{n} d \mu_{q}(y) \\
=\frac{[d]_{q^{\alpha}}^{n}}{[d]_{q}} \sum_{a=0}^{d-1} q^{a} \int_{\mathbb{Z}_{p}} \xi^{y}\left[\frac{x+a}{d}+y\right]_{q^{\alpha d}}^{n} \\
=\frac{[d]_{q^{\alpha}}^{n}}{[d]_{q}} \sum_{a=0}^{d-1} q^{a} \widetilde{\beta}_{n, \xi, q^{d}}^{(\alpha)}\left(\frac{x+a}{d}\right) .
\end{aligned}
$$

Therefore, by (21), we obtain the following theorem.

Theorem 8 For $n \in \mathbb{Z}_{+}, d \in \mathbb{N}$, and $\alpha \in \mathbb{Q}$, we have

$$
\widetilde{\beta}_{n, \xi, q}^{(\alpha)}(x)=\frac{[d]_{q^{\alpha}}^{n}}{[d]_{q}} \sum_{a=0}^{d-1} q^{a} \widetilde{\beta}_{n, \xi, q^{d}}^{(\alpha)}\left(\frac{x+a}{d}\right)
$$

From (9), we note that

$$
\begin{aligned}
& \widetilde{\beta}_{n, \xi^{-1}, q^{-1}}^{(\alpha)}(1-x) \\
& \quad=\int_{\mathbb{Z}_{p}} \xi^{-y}[1-x+y]_{q^{-\alpha}}^{n} d \mu_{q^{-1}}(y) \\
& =\frac{(-1)^{n} q^{\alpha n}}{\left(1-q^{\alpha}\right)^{n}} \sum_{l=0}^{n}\left(\begin{array}{l}
n \\
l
\end{array}\right)(-1)^{l} q^{\alpha l x-\alpha l} \int_{\mathbb{Z}_{p}} \xi^{-y} q^{-\alpha l y} d \mu_{q^{-1}}(y) \\
& =q^{\alpha n}(-1)^{n}\left(\frac{1}{\left(1-q^{\alpha}\right)^{n-1}} \sum_{l=0}^{n-1}\left(\begin{array}{l}
n \\
l
\end{array}\right)(-1)^{l} q^{\alpha l x} \frac{\alpha l+1}{1-\xi q^{\alpha l+1}}\right) \\
& =q^{\alpha n}(-1)^{n} \widetilde{\beta}_{n, \xi, q}^{(\alpha)}(x) .
\end{aligned}
$$


Therefore, by (22), we obtain the following theorem.

Theorem 9 For $n \in \mathbb{Z}_{+}$and $\alpha \in \mathbb{Q}$, we have

$$
\widetilde{\beta}_{n, \xi^{-1}, q^{-1}}^{(\alpha)}(1-x)=(-1)^{n} q^{\alpha n} \widetilde{\beta}_{n, \xi, q}^{(\alpha)}(x) .
$$

It is easy to show that

$$
\begin{aligned}
& \int_{\mathbb{Z}_{p}} \xi^{-x}[1-x]_{q^{-\alpha}}^{n} d \mu_{q^{-1}}(x) \\
& =\int_{\mathbb{Z}_{p}} \xi^{x}\left(1-[x]_{q^{\alpha}}\right)^{n} d \mu_{q}(x) \\
& =(-1)^{n} q^{\alpha n} \int_{\mathbb{Z}_{p}} \xi^{x}[x-1]_{q^{\alpha}}^{n} d \mu_{q}(x) .
\end{aligned}
$$

By (22) and (23), we obtain the following corollary.

Corollary 10 For $n \in \mathbb{Z}_{+}$and $\alpha \in \mathbb{Q}$, we have

$$
\begin{aligned}
\int_{\mathbb{Z}_{p}} \xi^{-x}[1-x]_{q^{-\alpha}}^{n} d \mu_{q^{-1}}(x) & =\sum_{l=0}^{n}\left(\begin{array}{c}
n \\
l
\end{array}\right)(-1)^{l} \widetilde{\beta}_{l, \xi, q}^{(\alpha)} \\
& =(-1)^{n} q^{\alpha n} \widetilde{\beta}_{n, \xi, q}^{(\alpha)}(-1)=\widetilde{\beta}_{n, \xi^{-1}, q^{-1}}^{(\alpha)}(2) .
\end{aligned}
$$

\section{Competing interests}

The author declares that they have no competing interests.

\section{Acknowledgement}

This paper was supported by Kon-Kuk University in 2012.

Received: 24 April 2012 Accepted: 1 August 2012 Published: 7 August 2012

\section{References}

1. Bayad, A: Modular properties of elliptic Bernoulli and Euler functions. Adv. Stud. Contemp. Math. 20, 389-401 (2010)

2. Bayad, A, Kim, T: Identities for the Bernoulli, the Euler and the Genocchi numbers and polynomials. Adv. Stud. Contemp. Math. 20, 247-253 (2010)

3. Carlitz, L: Expansion of q-Bernoulli numbers and polynomials. Duke Math. J. 25, 355-364 (1958)

4. Carlitz, L: q-Bernoulli numbers and polynomials. Duke Math. J. 15, 987-1000 (1948)

5. Cancul, IN, Kurt, V, Ozden, H, Simsek, Y: On the higher-order w- $q$-Genocchi numbers. Adv. Stud. Contemp. Math. 9, 39-57 (2009)

6. Ding, D, Yang, J: Some identities related to the Apostol-Euler and Apostol-Bernoulli polynomials. Adv. Stud. Contemp. Math. 20, 7-21 (2010)

7. Hegazi, AS, Mansour, M: A note on q-Bernoulli numbers and polynomials. J. Nonlinear Math. Phys. 13, 9-18 (2006)

8. Jang, LC, Kim, WJ, Simsek, Y: A study on the $p$-adic integral representation on $\mathbb{Z}_{p}$ associated with Bernstein and Bernoulli polynomials. Adv. Differ. Equ. 2002, Article ID 163217 (2002)

9. Jang, LC: A study on the distribution of twisted q-Genocchi polynomials. Adv. Stud. Contemp. Math. 19, 181-189 (2009)

10. Kim, T, Choi, J: On the q-Bernoulli numbers and polynomials with weight $\alpha$. Abstr. Appl. Anal. 2011, Article ID 392025 (2011)

11. Kim, T: 9 -Bernoulli numbers and polynomials associated with Gaussian binomial coefficients. Russ. J. Math. Phys. 15 51-57 (2008)

12. Kim, $\mathrm{T}$ : Some identities on the $q$-Euler polynomials of higher order and $q$-Stirling numbers by the fermionic $p$-adic integral on $\mathbb{Z}_{p}$. Russ. J. Math. Phys. 16, 484-491 (2009)

13. Kim, T: Barnes-type multiple q-zeta functions and q-Euler polynomials. J. Phys. A, Math. Theor. 43, Article ID 255201 (2010)

14. Kim, T: On a $q$-analogue of the $p$-adic log gamma functions and related integrals. J. Number Theory $76,320-329$ (1999) 
15. Kim, T: On the weighted $q$-Bernoulli numbers and polynomials. Adv. Stud. Contemp. Math. 21(2), 207-215 (1999)

16. Kupershmidt, BA: Reflection symmetries of q-Bernoulli polynomials. J. Nonlinear Math. Phys. 12, $412-422$ (2005)

17. Ozden, H, Cangul, IN, Simsek, Y: Remarks on $q$-Bernoulli numbers associated with Daehee numbers. Adv. Stud. Contemp. Math. 18, 41-48 (2009)

18. Rim, S-H, Jin, J-H, Moon, E-J, Lee, S-J: On multiple interpolation function of the $q$-Genocchi polynomials. J. Inequal. Appl. 2010, Article ID 351419 (2010)

19. Rim, S-H, Moon, E-J, Lee, S-J, Jin, J-H: Multivariate twisted $p$-adic $q$-integral on $\mathbb{Z}_{p}$ associated with twisted $q$-Bernoulli polynomials and numbers. J. Inequal. Appl. 2010, Article ID 579509 (2010)

20. Ryoo, CS: On the generalized Barnes type multiple $q$-Euler polynomials twisted by ramified roots of unity. Proc. Jangjeon Math. Soc. 13(2), 255-263 (2010)

21. Ryoo, CS: A note on the weighted q-Euler numbers and polynomials. Adv. Stud. Contemp. Math. 21, $47-54$ (2011)

22. Simsek, Y: Generating functions of the twisted Bernoulli numbers and polynomials associated with their interpolation functions. Adv. Stud. Contemp. Math. 16, 251-278 (2008)

23. Simsek, Y: On $p$-adic twisted $q$-L-functions related to generalized twisted Bernoulli numbers. Russ. J. Math. Phys. 13 340-348 (2006)

24. Simsek, Y: Theorems on twisted L-function and twisted Bernoulli numbers. Adv. Stud. Contemp. Math. 11, 205-218 (2005)

25. Simsek, Y: Special functions related to Dedekind-type sums and their applications. Russ. J. Math. Phys. 17, 495-508 (2011)

doi:10.1186/1029-242X-2012-176

Cite this article as: Jang: Some identities on the twisted $q$-Bernoulli numbers and polynomials with weight $\alpha$. Journal of Inequalities and Applications 2012 2012:176.

\section{Submit your manuscript to a SpringerOpen ${ }^{\circ}$ journal and benefit from:}

- Convenient online submission

- Rigorous peer review

- Immediate publication on acceptance

- Open access: articles freely available online

- High visibility within the field

- Retaining the copyright to your article 